\title{
A rare Bochdalek hernia in an adult: a case report
}

\author{
Yi-Min Gu ${ }^{\dagger}$, Xiao-Yang Li ${ }^{\dagger}$, Wen-Ping Wang and Long-Qi Chen * (1)
}

\begin{abstract}
Background: Symptomatic Bochdalek hernias are found mainly in infants in respiratory distress and occur rarely in adults.

Case presentation: We report a rare case of Bochdalek hernia associated with developmental abnormalities in an adult who exhibited acute chest pain and dyspnea on exertion.

Conclusions: This case highlights the importance of the differential diagnosis of acute left-sided chest pain and antenatal examination.

Keywords: Bochdalek hernia, Congenital diaphragmatic hernia, Adult, Chest pain, Anomaly of intestinal rotation, Patch repair
\end{abstract}

\section{Background}

Bochdalek hernia (BH) is one of the most common diaphragmatic abnormalities in infants and is characterized by cyanosis, tachycardia, and asymmetric growth in the chest cavity. By contrast, symptomatic $\mathrm{BH}$ is rare in adults. Reduction of the herniated content back into the peritoneal cavity, repair of the hernia, and relief of the intestinal obstruction are important objectives of operative repair. We describe here a case involving a 19-year-old woman with $\mathrm{BH}$ who presented with acute paroxysmal left chest pain radiating to the epigastrium. She underwent a mesh-based patch repair and the herniated viscera were returned to the abdominal cavity.

\section{Case presentation}

A 19-year-old woman presented with acute paroxysmal left chest pain radiating to the epigastrium of 4 days duration. She had a 10-year history of exertional dyspnea that was aggravated in the supine position, but no history of

\footnotetext{
${ }^{*}$ Correspondence: drchenlq@scu.edu.cn

†Yi-Min Gu and Xiao-Yang Li contributed equally to this work

Department of Thoracic Surgery, West China Hospital, Sichuan University,

No. 37, Guoxue Alley, Chengdu 610041, China
}

chest or abdominal trauma. She was misdiagnosed with a pulmonary abscess at another hospital, and the oral antibiotic treatment was ineffectual. Clinical examination on admission to our hospital revealed bowel sounds and decreased air entry in the left chest and that the abdomen appeared to be scaphoid. No obstructive gastrointestinal symptoms were observed. An X-ray revealed herniated loops of both the small and large intestines in the left hemithorax, which produced multiple lucent shadows and severely reduced lung space (Fig. 1, arrow). Computed tomography (CT) images showed that the bowel loops and fat passed through a defect in the posterolateral left hemidiaphragm (Fig. 2, arrow).

A left posterolateral thoracotomy was performed through the sixth intercostal space. The diaphragmatic defect was found along with herniation of the jejunum, ileum, ileocecal junction, appendix, and ascending and transverse colon. Pulmonary hypoplasia was also seen in the left lower lung lobe. We separated the adhesions around the foramen and within the pleural cavity. A laparotomy was subsequently performed through a median incision. During the operation, anomalies were found in intestinal rotation and fixation, and the total length of the 


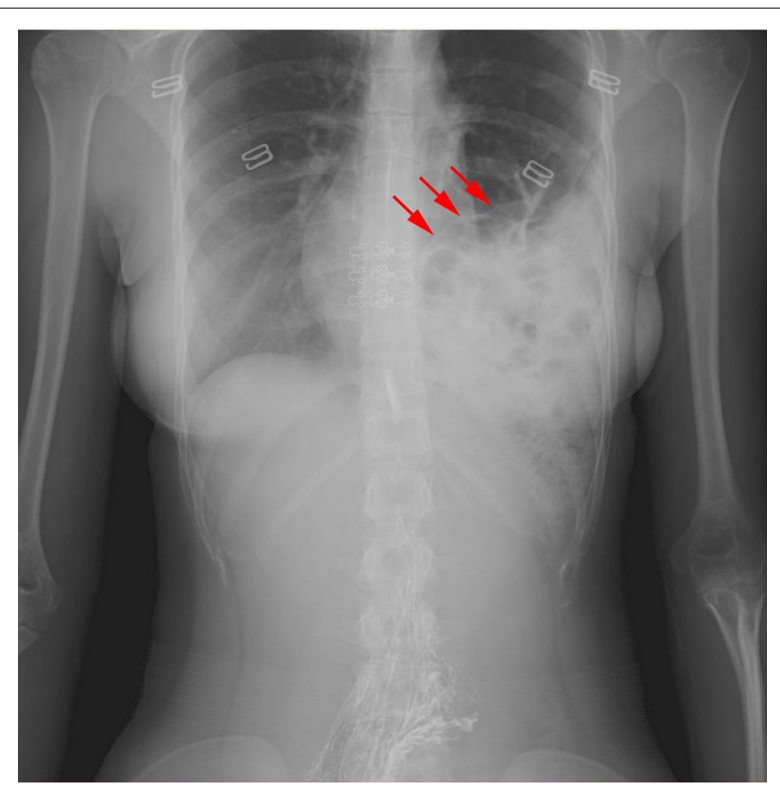

Fig. 1 An X-ray showing herniated loops in the left hemithorax, which appeared as multiple lucent shadows and severely reduced lung space (arrow)

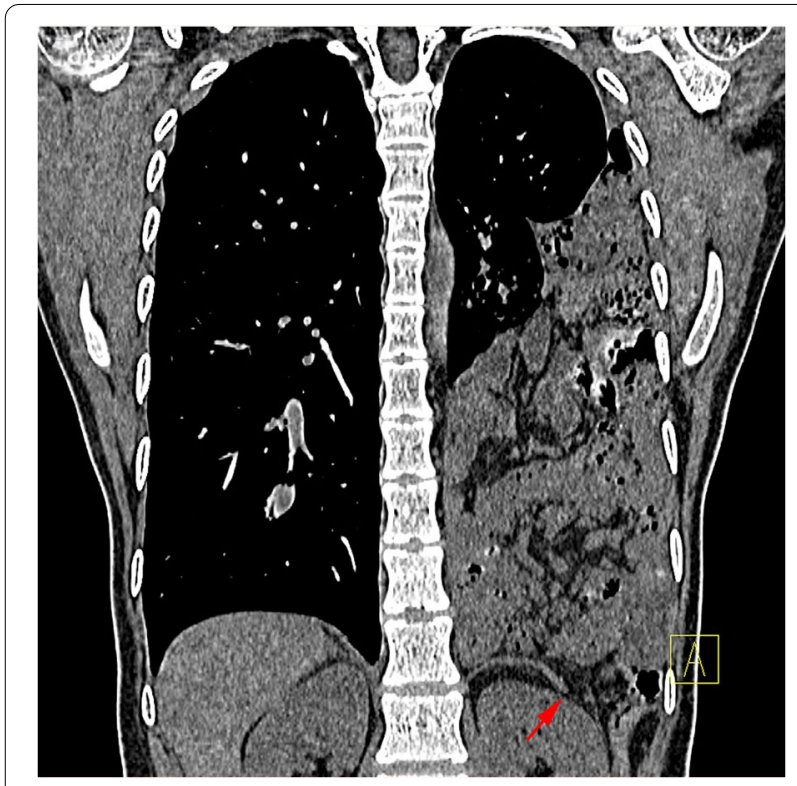

Fig. 2 Computed tomography images showing the bowel loops and fat passing through a defect in the posterolateral left hemidiaphragm (arrow)

jejunoileum was measured as $750 \mathrm{~cm}$. The herniated viscera were returned to the abdominal cavity.

We performed a sublay patch repair of the left posterolateral diaphragmatic hernia using a Dacron patch (Balance Medical Technology Co., Ltd., Beijing, China).
The procedure lasted for about $3 \mathrm{~h}$. The patient had an uneventful postoperative period. An X-ray of the upper gastrointestinal tract with soluble iodine contrast at the 1-month follow-up showed that the repair was satisfactory (Fig. 3). A chest X-ray revealed that the left upper lung lobe was expanded completely (Fig. 4). After the surgery, the patient's chest pain and dyspnea were relieved.

\section{Discussion and conclusions}

$\mathrm{BH}$ was first described by the Czech anatomist and pathologist, Vincent Alexander Bochdalek (1801-1883), in 1848 [1]. The condition is also known as a congenital posterolateral diaphragmatic hernia or pleuroperitoneal hernia. The abdominal viscera can herniate through into the thorax through the defect in the posterolateral part of the diaphragm. $\mathrm{BH}$ is one of the most common diaphragmatic abnormalities in infants and can cause respiratory distress in the newborn baby. The main clinical manifestations are cyanosis, tachycardia, and asymmetric growth in the chest cavity [2]. By contrast, $\mathrm{BH}$ is extremely rare in adults; its prevalence is $0.17-6 \%$ of all diaphragmatic hernias and it is almost always misdiagnosed.

Patients with $\mathrm{BH}$ can be asymptomatic or present with nonspecific gastrointestinal and respiratory signs and symptoms [3]. Other less common congenital hernias of the diaphragm in infants and children include hernias through the central tendon of the diaphragm, paraesophageal hernia, Morgagni hernia, and peritoneal pericardial hernia.

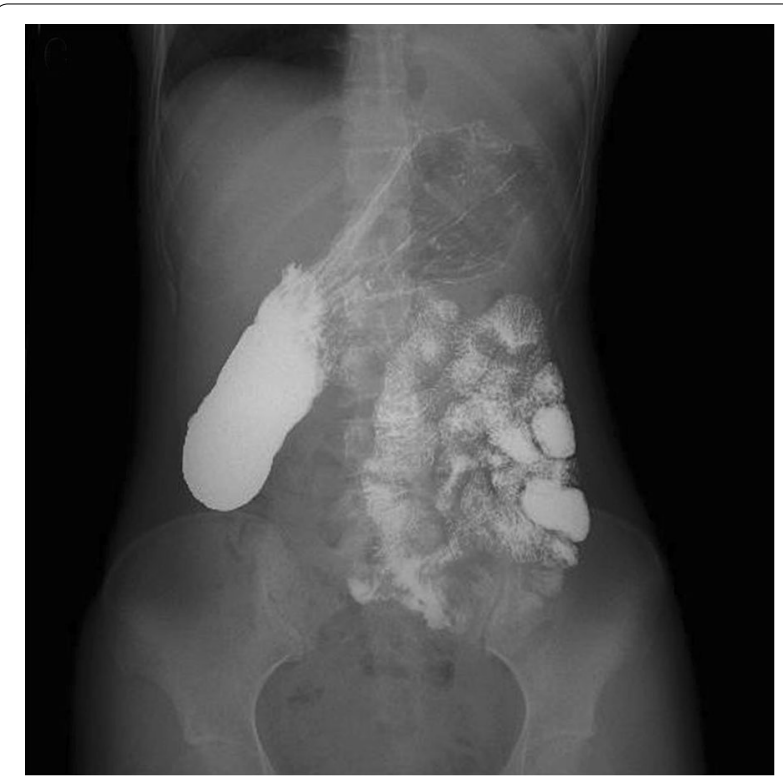

Fig. 3 X-ray with soluble iodine contrast at the 1-month follow-up showing the upper gastrointestinal tract and that the repair was satisfactory 


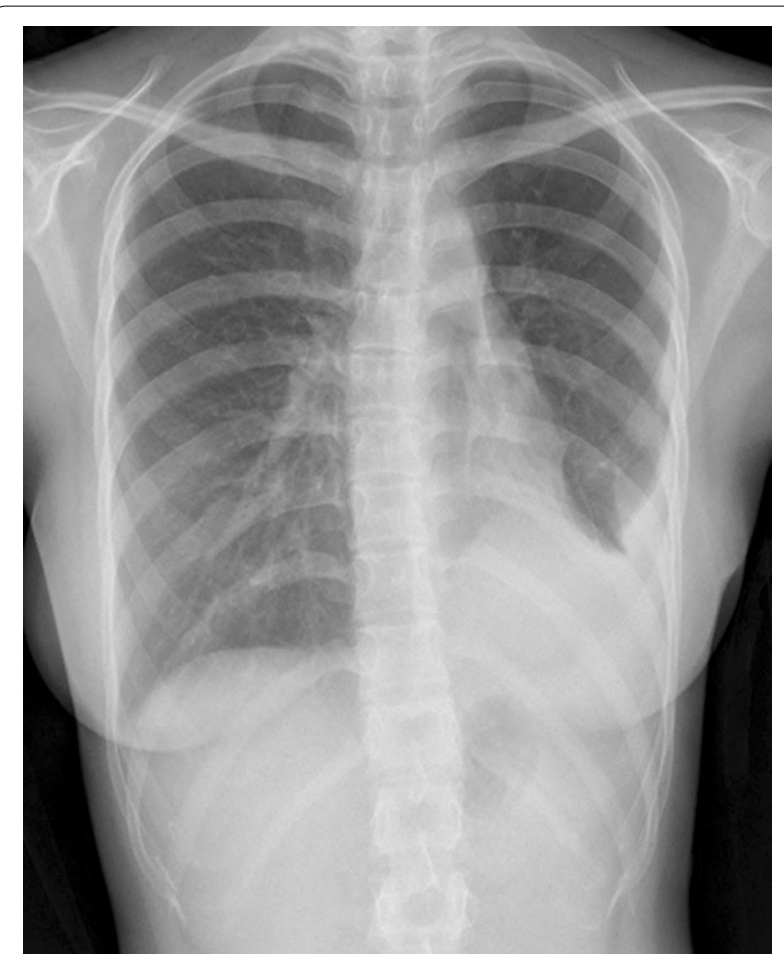

Fig. 4 Chest $X$-ray showing that the left upper lung lobe was completely expanded

Radiological examination has great clinical value for the discovery of $\mathrm{BH}$ and exclusion of other differential diagnoses, especially when $\mathrm{BH}$ is an incidental finding or is asymptomatic and involves a small congenital diaphragmatic hernia [4]. Radiological findings can identify diaphragmatic defects, congenital pulmonary malformations, and lesions occupying the intrathoracic space. Surgery is recommended to avoid future strangulation in both symptomatic and asymptomatic adult patients [5]. The primary option is the transabdominal approach, which has lower morbidity compared with thoracotomy. However, a laparoscopic or open laparotomy may not be the best choice for chronically incarcerated giant $\mathrm{BHs}$, in which extensive and severe adhesions are expected in the chest cavity. Therefore, the surgeon should choose an appropriate surgical method based on the situation of each patient.

We have described a rare case of a giant $\mathrm{BH}$ in an adult who presented with acute chest pain and dyspnea on exertion. The patient was initially misdiagnosed with a pulmonary abscess. After undergoing a mesh-based patch repair, the patient's pain and dyspnea were relieved, and good therapeutic outcomes were achieved.

\section{Abbreviations \\ BH: Bochdalek hernia; CT: Computed tomography.}

\section{Acknowledgements}

We want to thank our patient for consenting to the publication of the article.

\section{Authors' contributions}

GYM and LXY performed the literature review and formatted the manuscript. WWP participated in the diagnosis and treatment and supervised the writing of the article. CLQ performed the surgery and described the case. All authors read and approved the final manuscript.

\section{Funding}

Not applicable.

\section{Availability of data and materials}

All data generated or analyzed during this study are included in this published article.

\section{Declarations}

Ethics approval and consent to participate

Clearance was obtained from the Institutional Research and Ethics Review Committee of West China Hospital of Sichuan University for the publication of this case report.

\section{Consent for publication}

Written informed consent was obtained from the patient for publication of this case report and accompanying images.

\section{Competing interests}

Not applicable.

Received: 24 November 2020 Accepted: 23 September 2021

Published online: 12 October 2021

\section{References}

1. Hu X, Liu B. Bochdalek hernia. Lancet (Lond, Engl). 2018;392(10141):60.

2. Sarac M, Bakal U, Tartar T, Canpolat S, Kara A, Kazez A. Bochdalek hernia and intrathoracic ectopic kidney: presentation of two case reports and review of the literature. Niger J Clin Pract. 2018;21(5):681-6.

3. Tan CQY, Chan DL, Chu F, Fisher OM. Rare presentation of a Bochdalek hernia in adulthood with incarcerated splenic flexure of the colon mimicking diverticulitis: a report and review. ANZ J Surg. 2018;89:1518-20.

4. Lee J, Nam SH, Kim SW, Hong JM, Kim D. Diaphragmatic hernia with isolated shoulder pain evoked by surfeit. Ann Transl Med. 2019;7(1):11.

5. Davakis S, Kordzadeh A, Charalabopoulos A. An extraordinary rare cause of acute dyspnea in an adult. Gastroenterology. 2019;156(6):e15-6.

\section{Publisher's Note}

Springer Nature remains neutral with regard to jurisdictional claims in published maps and institutional affiliations. 